\title{
Placental stem cells, organotypic cul- ture and human placenta extract have neuroprotective activity in vitro
}

\author{
Prokopyuk V. Yu., Chub O. V., Shevchenko M. V., Prokopyuk O. S. \\ Institute for Problems of Cryobiology and Cryomedicine of the National Academy of Sciences of Ukraine, Kharkiv, Ukraine \\ e-mail: v.yu.prokopiuk@gmail.com
}

\section{ABSTRACT}

According to WHO, 6.7 million people die from stroke every year. The search for new neuroprotective substances remains an urgent task.

THE PURPOSE of this study was to investigate the neuroprotective activity of factors of placental origin.

MATERIALS AND METHODS. Neuroprotective activity of media conditioned with cryopreserved placenta derived mesenchymal stem cells (MSCs), organotypic culture of placenta and placental extract was studied on in vitro models of glutamate excitotoxicity in rats` neural cells. Neural cells were cultured with placental factors without glutamate treatment, before and after glutamate treatment. Neural cells' metabolic activity was assessed by MTT test.

RESULTS. Placental factors increase the MTT test indexes, prevent the toxic effect of glutamate on neural cells and promote their recovery. The thermolability of factors of placental origin and the effectiveness of various placental preparations are shown.

CONCLUSIONS. Conditional media of placenta derived MSCs, organotypic culture of the placenta and human placental extract have neuroprotective effect on rats' brain cells in vitro.

KEYWORDS: placenta; mesenchymal stem cells; placental extract; neural cells; neuroprotective effect

According to WHO, stroke is the second leading cause of death after cardiovascular disease, and more than 6 million people die from it every year. The contribution of strokes to the mortality structure has increased over the last 10 years, as they are among the least curable diseases. In addition, in the developed countries, senile dementia and demyelinating diseases are the fourth leading cause of death [17]. The urgent task of modern medicine is the search for new effective treatments that reduce damage, prevent death and contribute to the restoration of neurons and glia $[5,7,8]$. Numerous studies have shown that therapy with mesenchymal stem cells (MSCs), including placental origin, can be effective in diseases of the nervous system, including strokes and traumas $[3,5,19]$. In addition, the positive effect of tissue, cellular factors $[4,16]$, female sex hormones and placental extract on the nervous system was shown $[2,15,18]$. In this case, the mechanism of neuroprotective action of MSCs is associated with paracrine interactions, effects on neurons and glia [4, 8]. It can be assumed that the placenta derivatives, such as, stem cells as well as extract and explants (organotypic culture) will have neuroprotective and therapeutic effects. Placenta is one of the most accessible and promising sources of biomaterials, however, its application is impossible without creation of a low-temperature bank that provides the proper quantity and quality of preparations, moreover, cryopreservation changes the properties of the placental biomaterial [10-13].

The aim of this work was to study the neuroprotective activity of cryopreserved organotypic culture of placenta villi (explants), placenta derived mesenchymal stem cells and placental extract.

\section{MATERIALS AND METHODS}

Design of the experiment. To study the neuroprotective effect of placental factors, a model of glutamate excitotoxicity (damage of neural cells (NCs) due to neurotransmitter overexcitation), which takes place in the pathogenesis of a number of neurological diseases (ischemia, traumatic injuries, neurodegenerative diseases) and is the standard method for studying neuroprotective activity $[6,9]$ was chosen. The study was divided into 3 stages (Table 1). At the first stage, the effect of media conditioned with placental explants, placenta derived MSCs and media 
Table 1. Design of the experiment

\begin{tabular}{|l|c|c|c|}
\hline \multicolumn{1}{|c|}{ GROUP } & DAY 1 & DAY 2 & DAY 3 \\
\hline Positive control & - & - & MTT \\
\hline Negative control & - & Glu & MTT \\
\hline Stage 1 (effect on intact NCs) & - & Media & MTT \\
\hline Stage 2 (neuroprotective effect) & Media & Glu & MTT \\
\hline Stage 3 (regenerative effect) & Glu & Media & MTT \\
\hline
\end{tabular}

with the addition of $10 \%$ placental extract to NCs without glutamate was investigated. At the second stage, the neuroprotective (prophylactic) effect of the same media was investigated by NCs incubating with them for 1 day prior to glutamate treatment. At the third stage, the regenerating (curative) effect of substances on NCs after glutamate treatment was investigated. In all cases, both the effect of native media and media inactivated by heating to $90{ }^{\circ} \mathrm{C}$ for 30 minutes were investigated. The media conditioned with MSCs and placenta explants were used for 1 day according to the previously described protocol $[10,12]$, the concentration of placental extract was previously empirically selected and was $10 \%$ of the culture medium [1]. It was assumed that a day is enough for paracrine factors to enter the media, but is no time for media to deplete, or for saturating with the products of metabolism.

Placentas were obtained with the informed consent of women after cesarean section. The experiments were carried out in accordance with the "General principles of animal experiments» approved by the $V$ Congress on Bioethics (Kyiv, 2013) and the «European Convention for the Protection of Vertebrate Animals used for experimental and other scientific purposes» (Strasbourg, 1986), agreed with the Committee on Bioethics of the Institute of Problems of Cryobiology and Cryomedicine of the National Academy of Sciences of Ukraine (report No. 2 of 03.06.2013).

Culture of neural cells. NCs were isolated from the brain of newborn Wistar rats on the first day after their birth by a previously developed method [14]. For this purpose, the tissue was disaggregated for 15 minutes in $0.25 \%$ trypsin solution (BioWest, France) on phosphate buffered saline (PBS) at $37{ }^{\circ} \mathrm{C}$. The cells were cultured in 6-well plates (SPL, Korea) in DMEM with high content of glucose and L-glutamine (BioWest, France) supplemented with $10 \%$ fetal bovine serum (FBS) (Lonza, Germany) in a $\mathrm{CO}_{2}$ incubator at $37{ }^{\circ} \mathrm{C}$ in an atmosphere of $5 \% \mathrm{CO}_{2}$ In 5 days after adhesion, the non-adherent cells were removed by washing with PBS. The remaining cells were removed with a solution of $0.25 \%$ trypsin and Versene (EDTA) in a ratio of 1:1 and used in the glutamate excitotoxicity model. For this experiment, a culture containing various brain cells was chosen, since the mechanism of damage and regeneration is considered by several authors as processes of interaction of neurons with glia $[4,8]$.

Model of glutamate excitotoxicity. For modeling glutamate excitotoxicity, he NCs were transferred to a 96 well plate at a concentration of $2 \cdot 10^{4}$ cells/well for 1 day for attachment, then the medium was replaced with the fresh one, with glutamate (Sigma, USA) in a final concentration of $10 \mathrm{mM}$ and incubated for 1 day in the same conditions. To assess the metabolic activity of cells, MTT test was used. The wells were washed twice with PBS, the medium was changed to fresh. MTT (Sigma, USA) was added at a final concentration of $0.5 \mathrm{mg} / \mathrm{ml}$, incubated for 4 hours, after which the medium was carefully selected, and the formazan crystals were dissolved with $10 \%$ SDS solution on dimethyl sulfoxide (Sigma, France). The absorption was measured on plate spectrophotometer Utrao SM600 (China) at a wavelength of $570 \mathrm{~nm}$. Each experiment was repeated on three different cell cultures, eight samples were taken from each. At the same time, the MTT test results for cells without glutamate treatment was taken as $100 \%$.
Obtaining of placental cryoextract. Placental extract was obtained by the previously described method [13]. The human placenta, delivered within 3 hours after cesarean section surgery, was fragmented, 2 parts of PBS were added to one part of the placental tissue, three times cooled by immersion in liquid nitrogen and heated in a water bath at $37^{\circ} \mathrm{C}$, centrifuged at $1500 \mathrm{rpm}$, the supernatant was taken.

The obtaining of medium conditioned with cryopreserved placental explants. The placental explant-conditioned medium was prepared by the previously described method [12]. $10 \mathrm{mg}$ of cryopreserved placental explants were cultured in 24-well plates (SPL, Korea) for 1 day in $1 \mathrm{ml}$ of DMEM with high content of glucose and L-glutamine (BioWest, France) supplemented with $10 \% \mathrm{FBS}$ (Lonza, Germany) in a $\mathrm{CO}_{2}$ incubator at $37^{\circ} \mathrm{C}$ in $5 \% \mathrm{CO}_{2}$.

Obtaining of a medium conditioned with cryopreserved placenta derived mesenchymal cells. Placenta MSCs were obtained from fetal membranes by enzymatic method using $0.25 \%$ trypsin [10]. The cells were previously phenotyped, there are CD90, CD73, CD105-positive, CD34-negative, and they are able to differentiate in osteogenic, adipogenic and chondrogenic lines $[10,11]$. To obtain medium conditioned with cryopreserved MSCs, cells were thawed in a water bath at $37^{\circ} \mathrm{C}$. After reaching a monolayer about $1 \cdot 10^{6}$ per $5 \mathrm{ml}$ medium in $25 \mathrm{~cm}^{2}$ flask (SPL, Korea), the medium was changed, and cells were cultured for 1 day in DMEM with high content glucose and L-glutamine (BioWest, France) supplemented with $10 \% \mathrm{FBS}$ (Lonza, Germany) in a $\mathrm{CO}_{2}$ incubator at $37^{\circ} \mathrm{C}$ in an atmosphere of $5 \% \mathrm{CO}_{2}$.

Cryopreservation of placental cells and explants. Placental explants and MSCs of the 3-4 passages were cryopreserved according to the previously used program $[10,12]$, the effectiveness of which was shown $[10,11,12]$. As a cryoprotective medium we used DMEM with a high content of glucose and L-glutamine (BioWest, France) supplemented with $10 \%$ FBS (Lonza, Germany) and $10 \%$ dimethyl sulfoxide (Sigma, USA). Placental explants and MSCs were frozen in cryotubes (Nunc, USA) using containers Mr. Frosty ${ }^{\mathrm{TM}}$ Freezing Container (Thermo Fisher Scientific, USA) with isopropanol at a rate of $1^{\circ} \mathrm{C}$ to $-70^{\circ} \mathrm{C}$, followed by immersion in liquid nitrogen. They were thawed in a water bath at $37^{\circ} \mathrm{C}$

Statistical analysis of results. The results were statistically processed using the Mann-Whitney test with Past V. 3.15 (University of Oslo, Norway) software, the differences at $p<0.05$ were significant.

\section{RESULTS AND DISCUSSION}

At the first stage, the influence of media with placental factors on the metabolic activity of NCs was studied. After the addition of the medium conditioned with MSCs, the MTT value increased by more than $20 \%$. The medium with $10 \%$ placental extract increased MTT by $30 \%$, and the medium, conditioned with placental explants, increased it by more than $25 \%$. At the same time, as all these factors after inactivation by heating didn`t change NCs` metabolic activity (Fig. 1.). This result agrees with the literature data on the study of neuroprotective properties of tissue factors [16], where the hypothesis of the protein-peptide nature of substances affecting cells is given.

At the second stage, the neuroprotective effect of media containing placental factors was investigated by incubating NCs with them before glutamate treatment. It was found that the preliminary exposure of the medium with placental MSCs or $10 \%$ placental extract makes NCs insensitive to glutamate, while the same medium, conditioned with organotypic placental culture, significantly reduces the sensitivity of NCs to glutamate. The same media, inactivated by heating, have no protective properties (Fig. 2). 


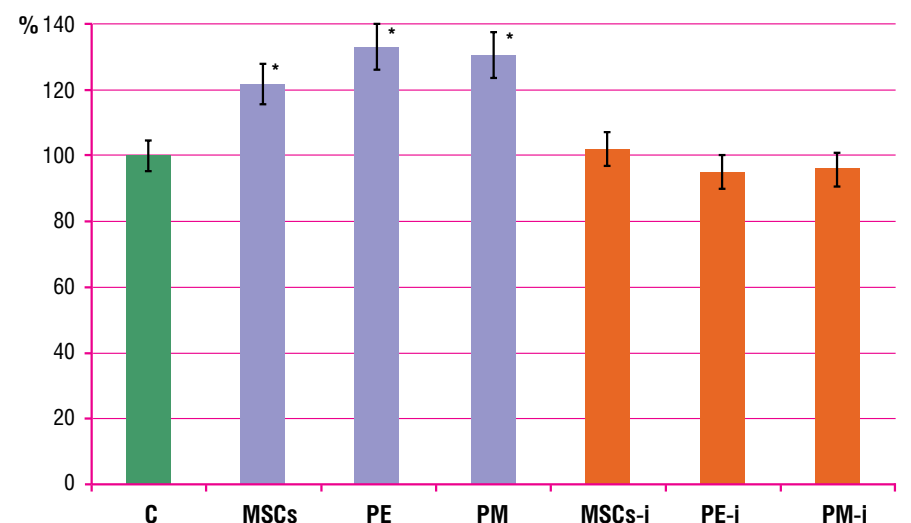

Fig. 1. Influence of factors of placental origin on the metabolic activity of neural cells according to the results of MTT test. C - control, neural cells without exposure; MSCs - neural cells with MSCs conditioned medium, PE - neural cells with $10 \%$ placental extract, $\mathrm{P}$ - neural cells with placental explants conditioned medium. MSCs-i, EP-i, P-i - the same media, inactivated by heating.

Note: ${ }^{*}-p<0.05$ compared with the control.

At the third stage of the study, the regenerative effect of placental factors on NCs culture was studied. It is shown that after glutamate treatment and subsequent regeneration in media containing placental factors, the metabolic activity of the cells is restored, but not more than $50 \%$ of the control value (fig. 3). In this case, the media, inactivated by heating, show no effect on NCs. There were no significant differences between media, containing placental extract and media conditioned with placenta derived MSCs or placental explants.

Thus, at all three stages of the experiment, it has been shown that placental factors increase NCs` metabolic activity according to MTT test, regardless of their source: as media conditioned by the cell culture or by the organotypic culture of the placenta and placenta extract. This confirms the opinion about the paracrine effect of MSCs on NCs. The results of the study are consistent with the literature data on the neuroprotective effect of MSCs [3, 5, 7, 8], placental $[3,15,19]$ and tissue factors [16]. It is shown that the neuroprotective effect is achieved by humoral action directly on NCs, and not on the whole organism $[5,7,8,15,19]$. Thermolability leads to the assumption that the effect is achieved due to substances of protein-peptide nature, and not estrogens, as shown by a number of researchers $[2,18]$. According to the experiments, we can not say about the advantages of these or other agents, since this requires the selection of concentrations that can differ for cell cultures, animal experiments, and clinical studies. At the same time, preventive use of placenta derivatives is more effective than their use after toxic effects, which can be regarded as neuroprotective action. It is advisable to consider derivatives of the placenta, primarily as a preventive and neuroprotective agent for NCs. In our opinion, it is promising to identify neuroprotective effects on different types of neural cells with subsequent detection of active substances and mechanisms of their effect at the next stages of the study.

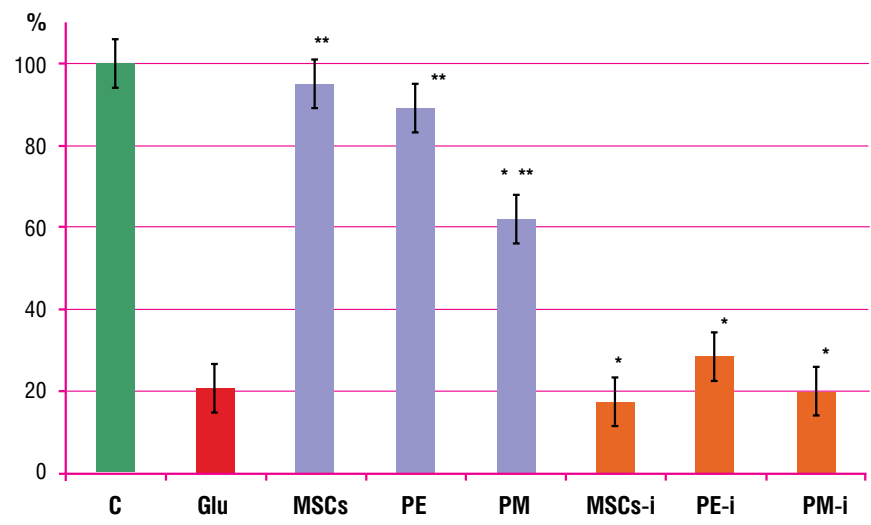

Aig. 2. The effect of preliminary incubation with the conditioned medium before $10 \mathrm{mM}$ glutamate treatment on neural cells metabolic activity by results of MTT test. C - control, neural cells without glutamate; Glu NCs after $10 \mathrm{mM}$ glutamate treatment; MSCs - neural cells after $10 \mathrm{mM}$ glutamate, with MSCs conditioned medium; PE - neural cells after $10 \mathrm{mM}$ glutamate, with $10 \%$ of placental extract medium, PM - neural cells after $10 \mathrm{mM}$ glutamate, with placental explants conditioned medium. MSCs-i, EP-i, PM- $-\mathrm{-}$ the same media, inactivated by heating.

Notes: * $-p<0.05$ compared to the control.

** $-p<0.05$ compared to 10 Glu.

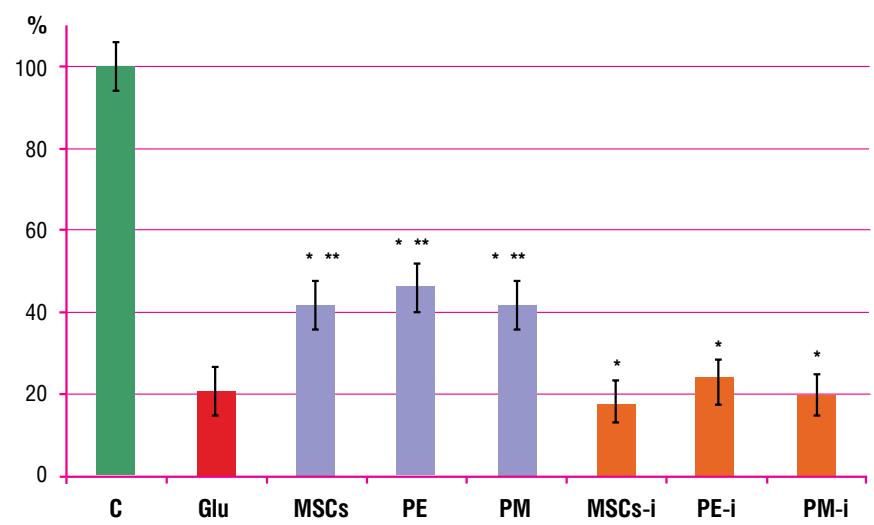

Fig. 3. The effect of conditioned media on the metabolic activity of neural cells after $10 \mathrm{mM}$ glutamate treatment according to the results of the MTT test. C - control, neural cells without glutamate; Glu - NCs after 10 mM glutamate treatment; MSCs - neural cells after $10 \mathrm{mM}$ glutamate, with conditioned medium of MSCs; PE - neural cells after $10 \mathrm{mM}$ glutamate, with $10 \%$ of placenta extract medium, PM - neural cells after $10 \mathrm{mM}$ glutamate, conditioned medium of placenta explants. MSCs-i, EP-i, PM-i the same media, inactivated by heating.

Notes: * $-p<0.05$ compared with the control,

** $-p<0.05$ compared to Glu group.

\section{CONCLUSIONS}

1. The media conditioned with placenta derived mesenchymal stem cells, placental explants, or enriched with placental extract, are characterized by neuroprotective activity in vitro.

2. Neuroprotective effect of media conditioned with placental mesenchymal stem cells, placental explants, or enriched with placental extract is more pronounced when they affect the neural cells culture prior to glutamate treatment than after glutamate toxicity.

3. Factors of placental origin, characterized by neuroprotective effects, are thermolabile. 


\section{REFERENCES}

1. Prokopyuk OS, Shevchenko NA, Prokopyuk VYu, et al. Vpliv kriokonservovanih bioob'ektiv placentarnogo pohodzhennja na kul'turu klitin [Influence of Cryopreserved Bioobjects of Placental Origin on Cell Culture]. Visnik problem biologiï i medicini - Bulletin of problems in biology and medicine. 2015; 1(122): 160-164. [In Ukrainian]

2. Benedusi V, Meda C, Sara D, et al. A lack of ovarian function increases neuroinflammation in aged mice. Endocrinology. 2012; 153(6): $2777-2788$.

3. Chen J, Shehadah A, Pal A, et al. Neuroprotective effect of human placenta-derived cell treatment of stroke in rats. Cell Transplant. 2013; 22(5): 871-879.

4. Cizkova D, Devaux S, Le Marrec-Croq F, et al. Modulation properties of factors released by bone marrow stromal cells on activated microglia: an in vitro study. Sci. Rep. 2014; doi:10.1038/srep07514

5. Gervois $P$, Wolfs $E$, Ratajczak J, et al. Stem cell-based therapies for ischemic stroke: preclinical results and the potential of imaging-assisted evaluation of donor cell fate and mechanisms of brain regeneration. Med Res Rev. 2016; 36(6): 1080-126.

6. Fukui $M$, Song JH, Choi J, et al. Mechanism of glutamate-induced neurotoxicity in HT22 mouse hippocampal cells. European Journal of Pharmacology. 2009; 619(1-3): $1-11$

7. Honmou O, Onodera R, Sasaki M, et al. Mesenchymal stem cells: therapeutic outlook for stroke. Trends Mol Med. 2012; 18(5): 292-97.

8. Hsuan YC, Lin CH, Chang CP, et al. Mesenchymal stem cell-based treatments for stroke, neural trauma, and heat stroke. Brain Behav. $2016 ; \mathbf{6}(\mathbf{1 0})$ : e00526.

9. Kanno H, Kawakami Z, Mizoguchi K, et al. Kampo medicine, protects PC12 cells from glutamate induced death by augmenting gene expression of cystine glutamate antiporter system. PLoS One. 2014; 9(12): e116275.

10. Pogozhykh D, Prokopyuk V, Pogozhykh O, et al. Influence of factors of cryopreservation and hypothermic storage on survival and functional parameters of multipotent stromal cells of placental origin. PLoS One. 2015; 10(10): 1-25.

11. Pogozhykh D, Pogozhykh O, Prokopyuk V, et al. Influence of temperature fluctuations during cryopreservation on vital parameters, differentiation potential, and transgene expression of placental multipotent stromal cells. Stem Cell Research \& Therapy. 2017; 8: 66.

12. Prokopyuk VYu, Prokopyuk OS, Musatova IB, et al. Safety of placental, umbilical cord and fetal membrane explants after cryopreservation. Cell and Organ Transplantology. 2015; 3(1): 34-38.

13. Schevchenko NO, Somova $K V$, Volina $V V$, et al. Dynamics of activity and duration of functioning of cryopreserved cryoextract, placental cells and fragments in the organism of experimental animals. Morphologia. 2016; 10(2): 93-98.

14. Sukach AN, Shevchenko MV, Liashenko TD. Comparative study on influence of fetal bovine serum and serum of adult rat on cultivation of newborn rat neural cells. Biopolymers and cell. 2014; 30(5): 394-99.

15. Takuma $K$, Mizoguchi $H$, Funatsu $Y$, et al. Placental extract improves hippocampal neuronal loss and fear memory impairment resulting from chronic restraint stress in ovariectomized mice. J Pharmacol Sci. 2012; 120: 89-97.

16. Wan Z, Mah D, Simtchouk S, et al. Human adipose tissue conditioned media from lean subjects is protective against H2O2 induced neurotoxicity in human SH-SY5Y neuronal cells. Int J Mol Sci. 2015; 16(1): 1221-31.

17. The top 10 causes of death. Available: http://www.who.int/mediacentre/factsheets/fs310/en/

18. Zhao L, Brinton RD. Select estrogens within the complex formulation of conjugated equine estrogens (Premarin $®$ ) are protective against neurodegenerative insults: implications for a composition of estrogen therapy to promote neuronal function and prevent Alzheimer's disease. BMC Neuroscience. 2006; 7: 24.

19. Zhi L, Zhao W, Liu W, et al. Transplantation of placenta-derived mesenchymal stem cell-induced neural stem cells to treat spinal cord injury. Neural Regen Res. 2014; 9(24): 2197-2204.

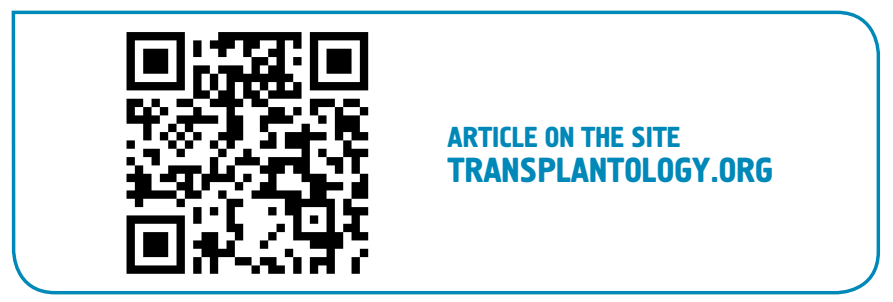

The authors indicate no potential conflicts of interest.

Received: November 17, 2016

Accepted: April 27, 2017

\section{SOURCE OF FUNDING.}

The work was carried out within Project No. 2.2.6.89 «Investigation of the geroprotective and geroterapeutic effect of placental bio-objects», Project No. 0113 U002955 «Genetic modification and long-term storage of placenta stem cells for clinical use», Project «Neuroprotective potential of cryopreserved placental MSCs, extract, serum of placental blood at damage to the spinal cord».

\section{ACKNOWLEDGMENTS.}

The authors would like to thank the colleagues from the Hannover Medical School of Medical University of Hannover (Germany) Dr. T. Mueller, Dr. D. Pogozhykh, Dr. O. Pogozhykh for their support and assistance with this research. 